\title{
Maximal sterile barrier precautions independently contribute to decreased central line-associated bloodstream Infection in infants with birth weight $\leq 1500$ gram: A Prospective Multicenter Observational Study
}

\author{
Daisuke Kinoshita, Yasushi Ohki, Satoshi Hada, Nobuaki Matsunaga, Haruyo Sakaki, Retsu Fujita \\ Neonatal Infection Control and Prevention Searching Group in Japan
}

\section{Introduction}

The specific elements of an effective central line-associated bloodstream infection (CLABSI) prevention bundle in the NICU remain unknown. The practice of PICC insertion in VLBWI remains diverse in Japan.

We aimed to investigate the risk factors for CLABSI in VLBWI.

\section{Results}

Total of 2383 infants with 33713 catheter-days were eligible for final analysis. From 302 suspected cases of CLABSI, we confirmed 70 cases, 35 as LCBI and 35 as CSEP. The overall rate of CLABSI was 2.1 per 1000 catheter-days.

Each NICU caregiver in Japan adopted various kinds of sterile precautions and that adherence to MSB was very low at $14 \%$.

Table 1. Demographics of the infants

\begin{tabular}{lc} 
Demographics & $\begin{array}{c}\text { total } \\
(\mathbf{n = 2 3 8 3})\end{array}$ \\
\hline Gestational age, wk & $\mathbf{2 8 . 5} \pm \mathbf{3 . 3}$ \\
Birth weight, $\mathbf{g}$ & $\mathbf{1 0 2 4} \pm \mathbf{3 0 1}$ \\
Female & $1126(47)$ \\
Apgar score at 5min & $7.2 \pm 2.0$ \\
Cesarean delivery & $1874(79)$ \\
Postnatal day upon PICC insertion, d & $1.7 \pm 2.7$ \\
\hline
\end{tabular}

Table 2.

Mixed effects Cox proportional hazard model analysis Variables Adjusted HR

\begin{tabular}{lcc} 
& $(95 \% \mathrm{Cl})$ & Pvalue \\
\hline MSB & $0.20(0.05-0.78)$ & .021 \\
Birth weight, $10 \mathrm{~g}$ & $0.98(0.967-0.999)$ & 0.038 \\
Type of skin antisepsis & & \\
$10 \%$ Povidone iodine & 1 (ref.) & \\
Less than $1 \%$ CHG/ethanol & $4.54(1.32-15.66)$ & .017 \\
\hline
\end{tabular}

The implementation of MSB, infant sex, gestational age, birth weight, five min Apgar score, mode of delivery, skin preparation, site of insertion, location of PICC insertion, experience of the physician, frequency of dressing change, and postnatal day of PICC insertion were included in the mixed effects Cox proportional hazard model, with neonatal center variable as the random effect. MSB implementation was associated with a lower CLABSI risk (adjusted HR $0.20 ; 95 \% \mathrm{Cl} 0.05-0.78$ ), whereas the use of $<1 \% \mathrm{CHG}$ was associated with a higher CLABSI risk (HR 4.54; 95\% Cl 1.32-15.66). (Table 2)

The Kaplan-Meier curve and the results of the log rank test showed that MSB implementation was associated with a lower cumulative incidence of CLABSI (crude HR 0.19; 95\% Cl 0.06-0.61). (Figure 1)

\section{Methods}

Design

Prospective multicenter observational study in Japan.

\section{Patients}

VLBWI and in whom a PICC was placed for the first time in 26 Japanese NICUs.

Period: Between October 2014 and March 2017.

\section{Primary endpoint}

CLABSI, including both laboratory confirmed bloodstream infection (LCBI) and clinical sepsis (CESP), within 28 days after PICC placement.

\section{Collection}

Information on PICC insertion and maintenance, including implementing maximal sterile barrier precautions (MSB),

Statistics

Risk factors of CLABSI, both related and unrelated to MSB, were assessed by the mixed effects Cox proportional hazard model.

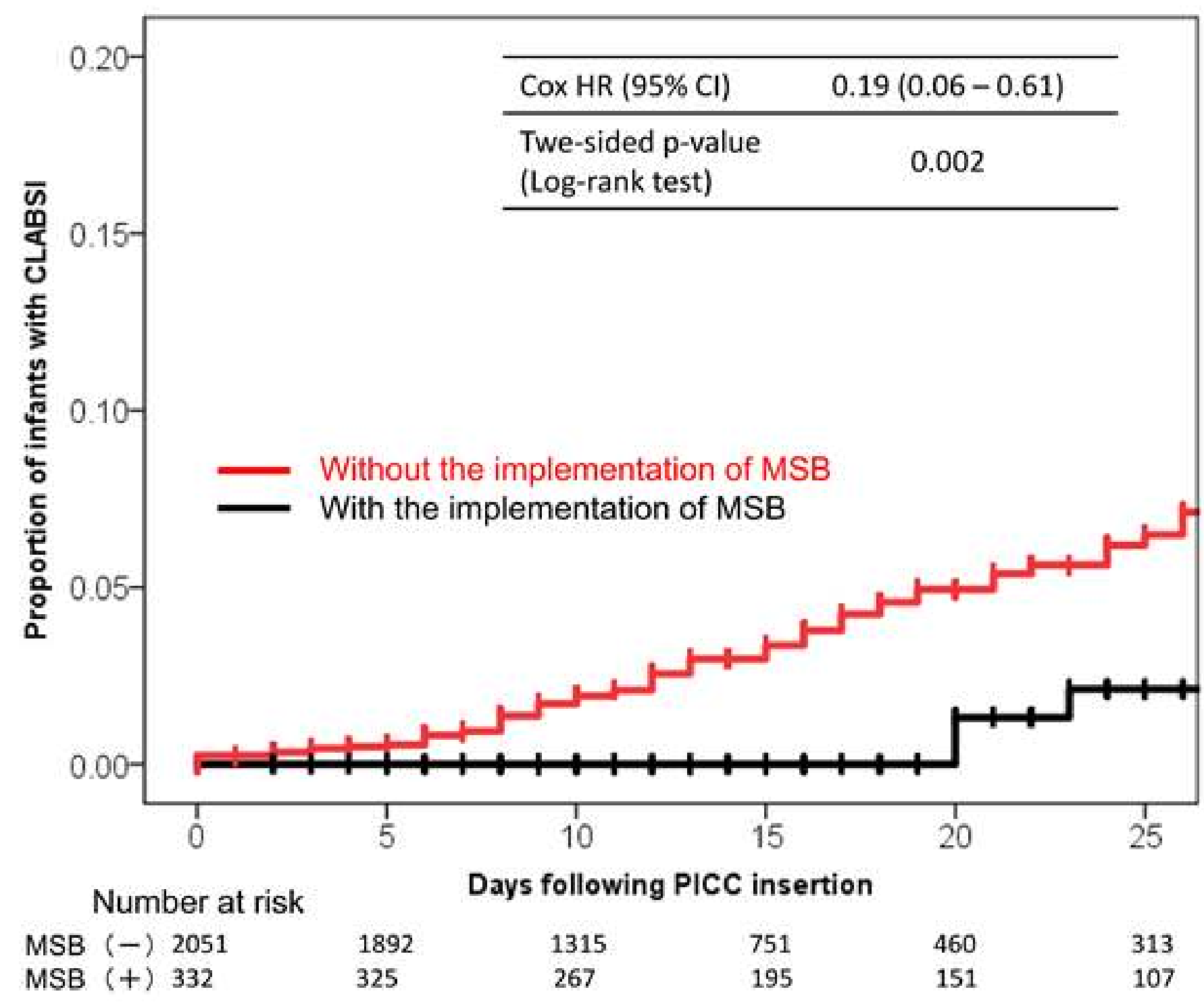

Figure 1.

Kaplan-Meier curve for the cumulative incidence of CLABSI

\section{Conclusion}

MSB implementation during PICC insertion in VLBWI was an independent factor that contributed to a decrease in CLABSI risk. 